\title{
Craniometria, morfometria do encéfalo e tipologia do circuito arterial da base do encéfalo em gatos*
}

\section{Craniometry, morphometry of brain and arterial circuit typology in brain cats}

\author{
Marcelo Salvador Gomes, ${ }^{* *}$ Sara Otoni da Silva Moraes, ${ }^{* * *}$ Carlos Augusto dos Santos Sousa, ${ }^{* * *}$ \\ Paulo de Souza Junior, ${ }^{* * * * *}$ Marcelo Abidu Figueiredo******
}

\begin{abstract}
Resumo
Objetivou-se descrever a tipologia do circuito arterial encefálico, a craniometria e a morfometria do encéfalo em gatos de ambos os sexos. As dissecções foram realizadas em 50 cadáveres de gatos adultos, 25 machos e 25 fêmeas, com média do comprimento rostro-sacral de $47,9 \mathrm{~cm}$ e $46,6 \mathrm{~cm}$, respectivamente. Após fixação com solução de formaldeído a $10 \%$ e preenchimento do sistema arterial com solução de látex corado procedeu-se a craniometria, craniotomia, remoção e morfometria dos encéfalos e dissecção para a observação da tipologia dos vasos responsáveis pela formação circuito arterial. Na presente pesquisa foi observado que, os machos apresentaram comprimento total do crânio, comprimento do crânio, largura do crânio e largura da face maior que as fêmeas. As fêmeas apresentaram o maior comprimento da face. Machos apresentaram maior índice da face enquanto que as fêmeas apresentaram maior índice do crânio. Não houve diferença nas medidas do cérebro e cerebelo. As artérias da base do encéfalo estiveram na dependência dos sistemas carótico e vértebro-basilar, responsáveis pela formação do circuito arterial do encéfalo. O padrão vascular encefálico dos gatos apresentou tendência do subtipo 2 alfa ao subtipo 2 beta, e encontrou-se entre os estágios médio e final de seu desenvolvimento filogenético.
\end{abstract}

Palavras-chave: crânio, encéfalo, medidas, vascularização.

\begin{abstract}
The aims of this study were to describe the typology of the arterial circuit of brain, craniometric measures and morphometry of the brain in cats of both sexes. The anatomical dissections were performed in 50 cadavers of adult cats, 25 male and 25 female, with a rostrum-sacral length of $47.9 \mathrm{~cm}$ and $46.6 \mathrm{~cm}$ respectively. After fixed with $10 \%$ formaldehyde solution and performing the injections with latex solution stained with pigment the craniometric measures, craniotomy, morphometry of the brain and dissection were made to identify the typology of the arterial circuit. In this study it was observed that males had total length of the skull, skull length, skull width and face width larger than the females. Females showed the greatest length of the face. Males had higher rates of the face while females had higher rates of the skull. There were no differences in measures of brain and cerebellum. The arteries of the base of the brain had been in the dependence of the carotid and vertebral-basilar systems, which are responsible for the brain arterial circuit formation. The cat's brain vascular pattern tended to be from subtype 2 alpha to beta 2 subtype, and considered among the middle and final stages of phylogenetic development.
\end{abstract}

Keywords: measurements, brain, skull, vascularization.

\section{Introdução}

O crânio é a parte mais complexa e especializada do esqueleto axial. Ele abriga o encéfalo e os órgãos sensoriais da audição, equilíbrio, visão, olfato e gustação, além dos órgãos dos sistemas respiratório e digestório. É basicamente dividido em uma região facial e uma neural. A forma e a morfometria dos crânios de carnívoros são parâmetros importantes para se determinar os diferentes tipos morfológicos (Evans, 2012).

Morfometria é o estudo científico das dimensões de estruturas anatômicas macroscópicas dos individuos, com especial referência às suas medidas lineares e pesos. Estas medidas são muitas vezes feitas por medições diretas em amostras fixadas em solução de formaldeído, especialmente no cérebro. Os resultados variam em precisão e ainda são motivos de discussão (Peters et al., 1998).

As doenças que acometem o sistema nervoso central dos animais domésticos representam grande parcela das afecções observadas na prática clinico-cirúrgica de animais de companhia; e podem ser responsáveis por deficiência de irrigação e consequente lesão isquêmica (Meneses, 2011).

*Recebido em 16 de abril de 2014 e aceito em 23 de março de 2015.

**Médico-veterinário autônomo, doutorando do Programa de Pós-graduação em Medicina Veterinária (Patologia e Ciências Clínicas) pela Universidade

Federal Rural do Rio de Janeiro , Seropédica, RJ - Brasil. e-mail: salvador-marcelo@hotmail.com.

***Médica-veterinária autônoma. e-mail:saraksmt@gmail.com.

****Médico-veterinário autônomo, mestrando do Programa de Pós-graduação em Biologia Animal pela Universidade Federal Rural do Rio de Janeiro, Seropédica, RJ - Brasil. e-mail: augusto.ar@hotmail.com.

*****Laboratório de Anatomia Animal da Universidade Federal do Pampa, campus Uruguaiana-RS. Rodovia BR-472, Km 592, C.P. 118, CEP 97500-970, Uruguaiana-RS. paulosouza@unipampa.edu.br.

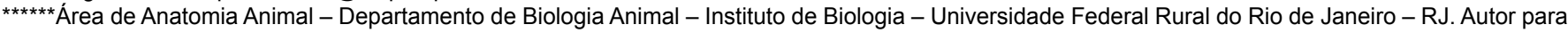
correspondência: marceloabidu@gmail.com. 
A abordagem cirúrgica desta região requer um conhecimento anatômico preciso, não só da habitual morfologia, mas também das variações das diversas estruturas que ali se encontram.

Em um estudo sobre a filogenia de artérias cerebrais, De Vriese (1905) classificou a irrigação encefálica de vertebrados em três tipos: Tipo I, no qual a irrigação cerebral é quase exclusivamente feita através das artérias carótida interna. Tipo II: no qual o encéfalo é parcialmente irrigado pela carótida e pelo sistema vertebrobasilar, com igual distribuição entre os dois sistemas ou a predominância de uma deles; e tipo III, no qual o encéfalo é irrigado quase exclusivamente através do sistema vertebrobasilar.

O estudo das artérias responsáveis pela irrigação do encéfalo nos animais domésticos e os que servem de modelo experimental considerando-se os aspectos filogenéticos, ainda é motivo de interesse na pesquisa biomédica devido à grande variabilidade dos arranjos apresentados pelas artérias formadoras do "círculo de Willis" (De Vriese, 1905, Testut, 1911, Barone \& Bortolami 2004, Prada, 2014), embora esses diferentes aspectos morfológicos não representem necessariamente parâmetros evolutivos (De Vriese, 1905). Dessa maneira, acredita-se que o comportamento das artérias encefálicas, comparativamente entre as diferentes espécies, exibe um modelo básico ao qual são acrescentadas modificações relativas aos diferentes grupos de animais (De Vriese, 1905, Testut, 1911).

Durante o contínuo processo de evolução do sistema nervoso central, foi possível observar a existência de constantes modificações na configuração dos vasos responsáveis por sua vascularização (Lima et al., 2006). Nesse contexto, verificou-se que ramos arteriais não supriram somente aquelas estruturas que originaram as suas denominações, mas também participaram significantemente do suprimento sanguíneo de outras estruturas importantes (Cunha et al., 2001). Objetivou-se com esta pesquisa efetuar a craniometria e morfometria do encéfalo de gatos caracterizando o tipo predominante do arranjo vascular do circuito arterial da base do encéfalo.

\section{Material e métodos}

Todos os cadáveres utilizados foram cedidos à área de Anatomia Animal, congelados e armazenados em freezer desde que foram recebidos. Foram utilizados 50 gatos, SRD, adultos, sendo 25 machos e 25 fêmeas. Este trabalho foi realizado no período de abril de 2011 a janeiro de 2012. Inicialmente os cadáveres foram descongelados em água corrente, sexados e identificados através da colocação de etiqueta plástica numerada fixada com barbante ao tendão calcâneo comum. Com o auxílio de uma trena metálica de precisão foi mensurado o comprimento rostrossacral de cada animal, tendo como referência a extremidade do focinho até a inserção da cauda.

Os cadáveres foram posicionados em decúbito lateral direito para acesso a porção torácica da aorta, através de incisão realizada entre o $6^{\circ}$ e o $10^{\circ}$ espaço intercostal. A artéria foi canulada com sonda uretral número oito ou dez (variável em função do diâmetro do vaso), sendo presa com barbante para evitar extravasamentos e manter a pressão intravascular. A fixação foi feita injetando-se, através da sonda, solução de formaldeído a 10 \% (volume variável em função do peso do cadáver) em sentido cranial e caudal.
Imediatamente após a fixação dos cadáveres, foi injetada solução de Petrolátex S65 (Refinaria Duque de Caxias - REDUCPetrobras/ Duque de Caxias/ RJ) corado com pigmento Suvinil para preenchimento de todo o sistema arterial. Em seguida os cadáveres foram imersos em caixas de polietileno de baixa densidade de capacidade de 500 litros contendo solução de formaldeído a 10 \% para a finalização do processo de fixação e polimerização do látex. Decorridos sete dias da injeção do látex, os cadáveres foram lavados em água corrente e dissecados. Antes da remoção dos encéfalos foram feitas medidas craniométricas utilizando-se como referência alguns acidentes ósseos na superfície da cabeça de acordo com Evans (2012) e adaptada para a espécie em estudo, como expresso na Figura 1, para obtenção das medidas lineares observadas na Figura 2.

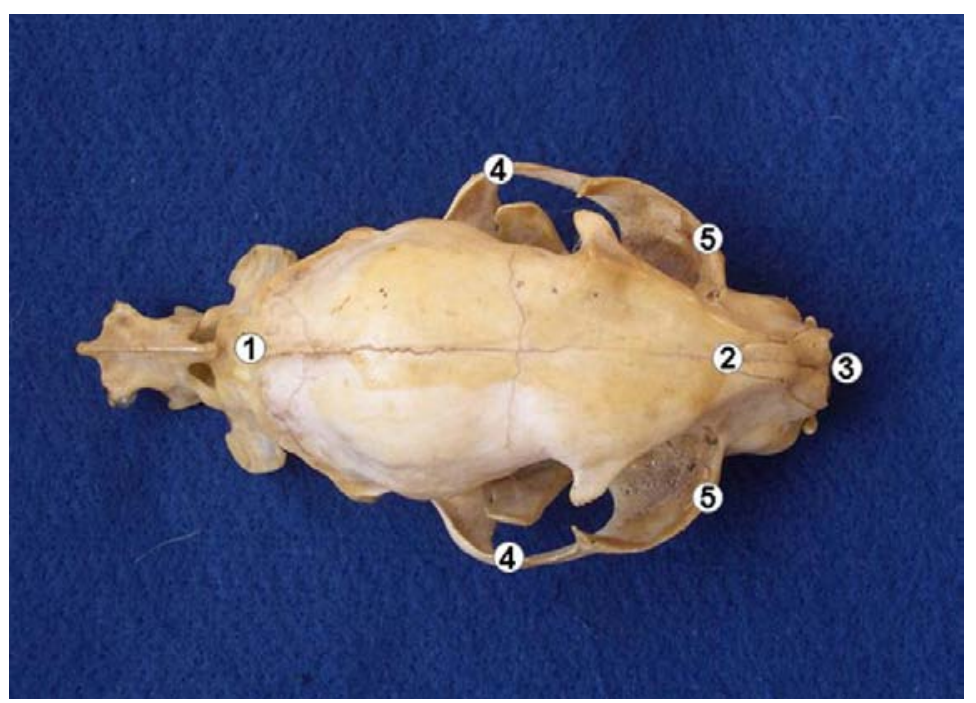

Figura 1: Fotomacrografia de pontos craniométricos utilizados como referência 1 = Íneo 2 = Násio 3 = Próstio 4 = arco zigomático (porção caudal) 5 = arco zigomático (porção rostral)

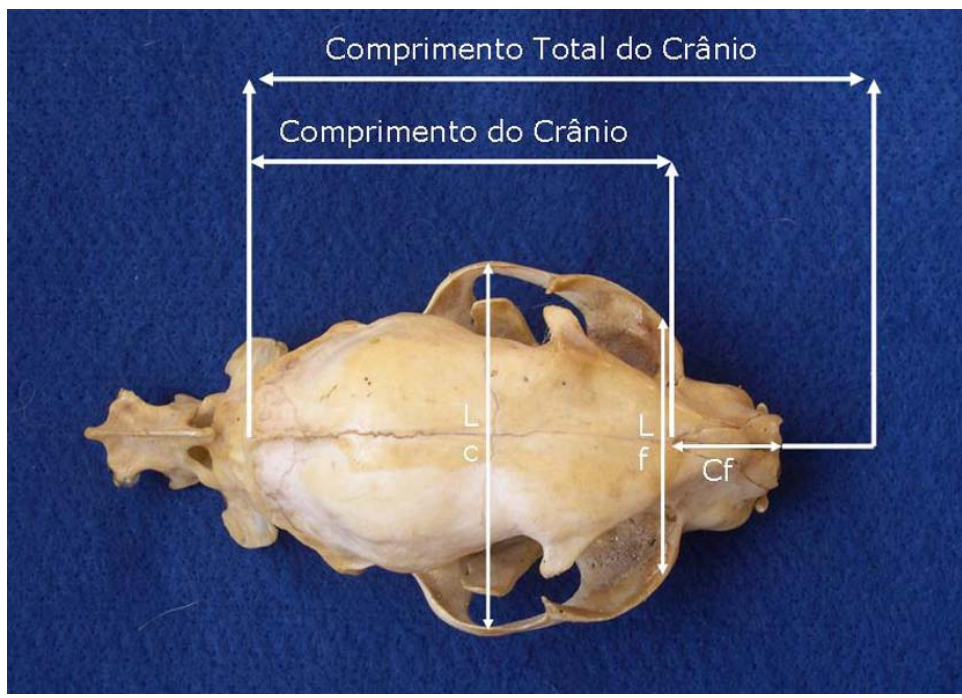

Figura 2: Fotomacrografia mostrando as medidas lineares obtidas $\mathrm{CF}=$ comprimento da face, $\mathrm{CC}=$ comprimento do crânio,

$\mathrm{CT}=$ comprimento total, LF = largura da face e LC = largura do crânio 
Também foram obtidos os índices do crânio (largura do crânio X100 / comprimento do crânio) e o da face (largura da face X100 / comprimento da face)

Depois de realizadas as medidas externas no crânio, com auxílio de bisturi, pinças oftálmicas e fórceps odontológico, foram retirados os encéfalos por meio de craniotomia. Cada encéfalo foi previamente identificado através de colocação de etiqueta plástica numerada fixada na extremidade da medula espinhal. Todas as medidas foram realizadas pelo mesmo observador. rostrossacral, optou-se por calcular o coeficiente de correlação "r". Todo o processamento dos dados foi realizado no Software Grafpad Prism 5.

Este experimento faz parte do projeto intitulado ANATOMIA VASCULAR NO GATO: ESTUDO DESTINADO À PRÁTICA CIRÚRGICA, EXPERIMENTAL E AO DIAGNÓSTICO POR IMAGEM, que foi submetido e aprovado pelo comitê de ética e pesquisa da Universidade Federal Rural do Rio de Janeiro, sob o processo de número 23083.005334 / 2009 - 49.

Com auxílio de um paquímetro de precisão foram obtidas as seguintes medidas do encéfalo:

$\mathrm{CHCD}=$ comprimento do hemisfério cerebral direito (medida obtida tendo como referência a distância entre os polos occipitais e frontais direitos).

$\mathrm{CHCE}=$ comprimento do hemisfério cerebral esquerdo (medida obtida tendo como referência a distância entre os polos occipitais e frontais esquerdos).

$\mathrm{LHCD}=$ largura do hemisfério cerebral direito (medida obtida tendo como referência à distancia entre o polo temporal direito e a fissura longitudinal do cérebro).

LHCE = largura do hemisfério cerebral esquerdo (medida obtida tendo como referência à distância entre o polo temporal esquerdo e a fissura longitudinal do cérebro).

LLPD= largura do lobo piriforme direito (medida obtida na porção mais dilatada do lobo piriforme direito).

LLPE = largura do lobo piriforme esquerdo (medida na porção mais dilatada do lobo piriforme esquerdo).

$\mathrm{Ccb}=$ comprimento do cerebelo (medida obtida tendo com referência à distância compreendida entre a porção rostral do cerebelo e a pirâmide do vermis).

LC= largura do cerebelo (medida obtida tendo como referência a distância entre as extremidades dos hemisférios cerebelares direito e esquerdo). Todos os encéfalos foram fotografados com câmera digital. As medidas foram obtidas sempre pelo mesmo observador.

Foram calculadas a média e o desvio padrão das medidas do crânio, do encéfalo e do animal. Essas medidas foram comparadas entre os antímeros e entre os sexos através do teste "t" não pareado considerando um $p<0,05$ como significativo. Em relação ao comportamento do conjunto: comprimento e largura do crânio e medidas do encéfalo, em função do comprimento

\section{Resultados}

Os resultados da craniometria, morfometria do encéfalo e correlação linear simples, estão representados nas Tabelas 1 e 2, respectivamente.

O arranjo geral das artérias da base do encéfalo dos machos e fêmeas está na dependência dos sistemas carótico e vertebrobasilar e é estabelecido a partir de vasos que, mediante suas divisões, em número e disposição variáveis, expressam diferentes significados estruturais e funcionais. A disposição e o arranjo apresentado pelas artérias da base do encéfalo dos gatos caracterizam o circuito arterial do encéfalo, que é visto circundando o corpo mamilar, a fossa interpenduncular, o túber cinéreo, a hipófise e o quiasma óptico. Este circuito arterial estende-se desde a ponte, até a porção rostral do quiasma óptico e apresentaram tendência ao subtipo 2 alfa ao subtipo 2 beta (Figura 3 ). 
Tabela 2: Valores da correlação linear simples entre a medida do comprimento rostrossacral, comprimento total do crânio, comprimento do crânio e as medidas do encéfalo em machos e fêmeas

\begin{tabular}{|c|c|c|}
\hline \multirow{2}{*}{ Correlação } & \multicolumn{2}{|c|}{ Gatos } \\
\hline & Machos & Fêmeas \\
\hline \multirow{3}{*}{ Comprimento total do crânio $\mathrm{X}$ Comprimento do Hemisfério Cerebral Esquerdo } & $P=0,3355$ & $P=0,7443$ \\
\hline & $r=-0,2054$ & $r=0,06867$ \\
\hline & $r^{2}=0,04220$ & $r^{2}=0,004716$ \\
\hline \multirow{3}{*}{ Comprimento total do crânio $X$ Comprimento do Hemisfério Cerebral Direito } & $P=0,5726$ & $P=0,9339$ \\
\hline & $r=0,1212$ & $r=-0,01747$ \\
\hline & $r^{2}=0,01469$ & $r^{2}=0,0003053$ \\
\hline \multirow{3}{*}{ Comprimento do crânio X Comprimento do Hemisfério Cerebral Direito } & $P=0,6947$ & $P=0,8537$ \\
\hline & $r=0,08447$ & $r=-0,03884$ \\
\hline & $r^{2}=0,007135$ & $r^{2}=0,001509$ \\
\hline \multirow{3}{*}{ Comprimento do crânio $\mathrm{X}$ Comprimento do Hemisfério Cerebral Esquerdo } & $P=0,3394$ & $P=0,9779$ \\
\hline & $r=0,2054$ & $r=0,005843$ \\
\hline & $r^{2}=0,04220$ & $r^{2}=3,414705$ \\
\hline \multirow{3}{*}{ Comprimento rostrossacral $\mathrm{X}$ Comprimento do Hemisfério Cerebral Direito } & $P=0,4198$ & $P=0,3263$ \\
\hline & $r=0,1727$ & $r=0,2047$ \\
\hline & $r^{2}=0,02981$ & $r^{2}=0,04190$ \\
\hline \multirow{3}{*}{ Comprimento rostrossacral X Comprimento do Hemisfério Cerebral Esquerdo } & $P=0,9644$ & $P=0,2886$ \\
\hline & $r=-0,009621$ & $r=0,2209$ \\
\hline & $r^{2}=9,25705$ & $r^{2}=0,04880$ \\
\hline \multirow{3}{*}{ Comprimento rostrossacral $X$ Largura do Lobo Piriforme Direito } & $P=0,0057$ & $P=0,3987$ \\
\hline & $r=0,5464$ & $r=0,1765$ \\
\hline & $r^{2}=0,2985$ & $r^{2}=0,03115$ \\
\hline \multirow{3}{*}{ Comprimento rostrosacral X Largura do Lobo Piriforme Esquerdo } & $P=0,9601$ & $P=0,2508$ \\
\hline & $r=0,01078$ & $r=0,2386$ \\
\hline & $r^{2}=0,0001163$ & $r^{2}=0,05692$ \\
\hline \multirow{3}{*}{ Comprimento rostrossacral $\mathrm{X}$ Largura do Cerebelo } & $P=0,5459$ & $P=0,4761$ \\
\hline & $r=0,1297$ & $r=0,1494$ \\
\hline & $r^{2}=0,01682$ & $r^{2}=0,02231$ \\
\hline \multirow{3}{*}{ Comprimento rostrossacral $X$ Comprimento do cerebelo } & $P=0,9122$ & $P=0,1610$ \\
\hline & $r=-0,02376$ & $r=0,2891$ \\
\hline & $r^{2}=0,0005647$ & $r^{2}=0,08360$ \\
\hline \multirow{3}{*}{ Comprimento rostrossacral X Largura do Hemisfério Cerebral Direito } & $P=0,8552$ & $P=0,1502$ \\
\hline & $r=-0,03935$ & $r=0,2964$ \\
\hline & $r^{2}=0,001548$ & $r^{2}=0,08785$ \\
\hline \multirow{3}{*}{ Comprimento rostrossacral X Largura do Hemisfério Cerebral Esquerdo } & $P=0,4002$ & $P=0,3721$ \\
\hline & $r=0,1799$ & $r=-0,1865$ \\
\hline & $r^{2}=0,03238$ & $r^{2}=0,03478$ \\
\hline \multirow{3}{*}{ Comprimento rostrossacral $\mathrm{X}$ Comprimento da face } & $P=0,6639$ & $P=0,1552$ \\
\hline & $r=-0,09349$ & $r=0,2930$ \\
\hline & $r^{2}=0,0087$ & $r^{2}=0,0588$ \\
\hline
\end{tabular}




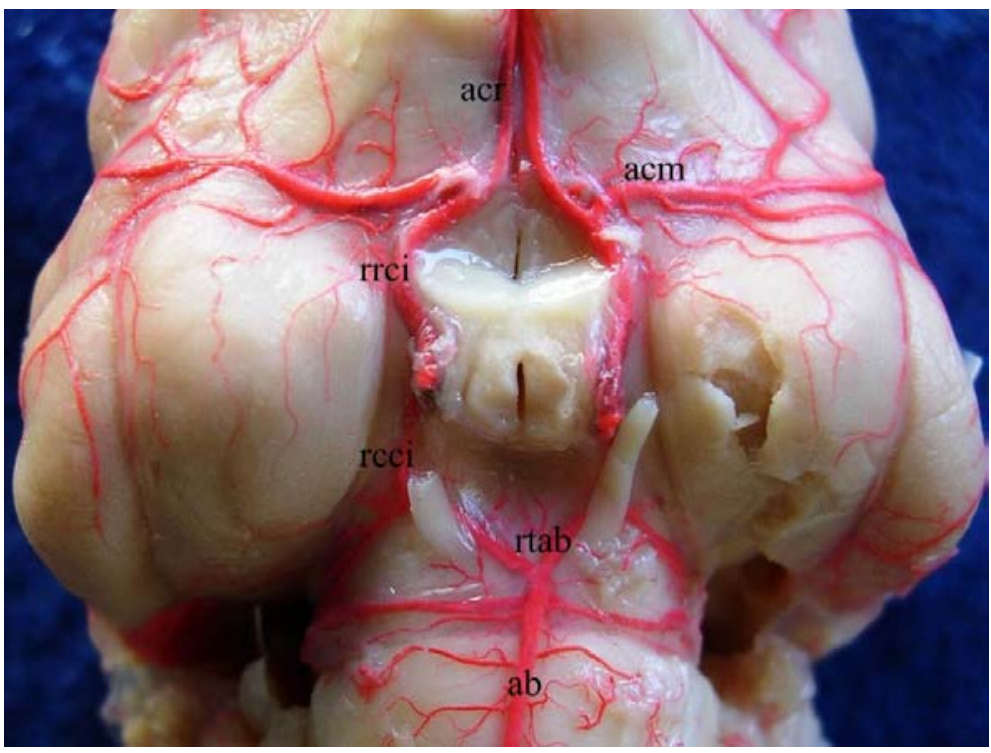

Figura 3: Fotomacrografia da vista ventral do encéfalo de gato. acr - artéria cerebral rostral, acm - artéria cerebral média, rrci - ramo rostral da carótida interna, rcci - ramo caudal da carótida interna, rtab - ramo terminal da artéria basilar, $\mathrm{ab}$ - artéria basilar.

\section{Discussão}

O crânio de cães e gatos mostra considerável variação individual e racial na forma e tamanho. A forma do crânio é critério importante na determinação do padrão racial de cães e gatos, e os índices de crânio são eficazes na separação ou definição dos tipos morfológicos (Evans, 2012).

Por esta razão, a forma do crânio de cães foi estudada por Brehm et al. (1985) e Regedon et al. $(1991,1992)$ e foi considerada nas pesquisas anatômicas de Zietzschmann (1943), Getty (1986) e Evans (2012) .

A aparência fenotípica da cabeça do gato depende da forma do crânio e está fortemente relacionada com a raça específica e as características do esqueleto (Künzel, 2003).

Na presente pesquisa foi observado que os machos apresentaram comprimento total do crânio, comprimento do crânio, largura do crânio e largura da face maiores que as fêmeas. As fêmeas apresentaram o maior comprimento da face. Machos apresentaram maior índice da face enquanto as fêmeas apresentaram maior índice do crânio.

As correlações entre a forma e os parâmetros de dimensão para o cérebro e crânio e as mudanças decorrentes da idade foram encontradas em estudo realizado por Onar (1999) em cães da raça Pastor Alemão. Na presente investigação não houve correlação em nenhum dos parâmetros avaliados.

Al-Sagair e El Mougy (2002) realizaram craniometria em camelos machos jovens e adultos e concluíram que todas as medidas aumentaram com a idade e apenas o índice craniano diminuiu. Na presente pesquisa, embora utilizando apenas animais adultos, foram observados, nos machos, maior índice da face enquanto as fêmeas apresentaram maior índice do crânio.
Olopade et al. (2005) realizaram a morfometria do encéfalo de ovinos separados por sexo, peso e idade e concluíram que não houve diferença nos parâmetros do encéfalo em relação ao sexo e a idade; entretanto, animais mais pesados apresentaram aumento no comprimento do encéfalo. Na presente investigação também não houve diferença nas medidas do encéfalo entre os sexos, com exceção da largura do hemisfério esquerdo que é maior nos machos quando comparado com o hemisfério esquerdo das femeas.

Ribeiro et al. (2005) realizaram morfometria em 81 hemisférios cerebrais humanos comparando os dois sexos e concluíram que os homens apresentaram maior comprimento e maior peso cerebral, diferente em parte dos resultados obtidos na presente investigação, na qual a comparação entre a largura dos hemisférios esquerdo entre os sexos, os machos apresentaram valores maiores.

Igaido e Aina (2010) em estudo referente às medidas do cérebro de codorna (Coturnix coturnix japonica) usando 20 machos e 15 fêmeas, observaram que as fêmeas apresentaram média dos valores de comprimento, peso e profundidade do cérebro, superiores aos machos. Esses resultados diferem dos obtidos em gatos de ambos os sexos dissecados na presente investigação, pois os machos apresentaram valores maiores para a largura do hemisfério esquerdo quando comparados aos das femeas.

Portugal et al. (2014) realizaram morfometria no encéfalo de coelhos da raça Nova Zelandia de ambos os sexos e verificaram que os machos apresentaram hemisférios cerebrais direitos mais largos do que os das femeas, diferente do observado na presente pesquisa, na qual machos apresentaram hemisférios cerebrais esquerdos mais largos do que os das femeas.

Em estudo realizado sobre a morfometria do encéfalo de equinos mestiços de ambos os sexos, Moraes et al. (2014) observaram que as femeas apresentaram os maiores valores de largura dos hemisferios cerebrais direito e esquerdo e tambem o comprimento do cerebelo. Em gatos de ambos os sexos dissecados na presente investigação, machos apresentaram hemisférios cerebrais esquerdos mais largos do que os das femeas.

Samuel et al. (2014) realizaram morfometria do encéfalo do gambá (Didelphys marsupialis cancrivora) de ambos os sexos e observaram que as fêmeas apresentaram os maiores valores, diferente do observado nos gatos da presente investigação no qual a comparação entre a largura dos hemisférios esquerdo entre os sexos, os machos apresentaram valores maiores.

De acordo com a classificação morfológica proposta por De Vriese (1905) para o estudo das artérias da base do encéfalo, foi observado que os gatos do presente estudo, apresentaram tendência ao subtipo 2 alfa ao subtipo 2 beta, e encontramse entre os estágios médio e final de seu desenvolvimento filogenético, conforme o considerado por Testut (1911). Nesta mesma categoria (entre os subtipos 2 alfa e 2 beta) Alcântara e Prada (1996 a b), Lindemann (2000) , Lima et al. (2006) e Prada (2014) classificaram o arranjo vascular encontrado respectivamente para cães, gambá e gato. 
Em relação aos caracteres gerais de diferenciação ontogenética e filogenética das artérias encefálicas, Testut (1911) afirma que primitivamente a artéria carótida interna é a única artéria que conduz sangue à massa encefálica; a artéria vertebral não existe. No estágio médio, as duas artérias cerebrais anteriores, até então independentes, unem-se na linha média, ora por meio de uma rede intermediária, ora por meio de um simples ramo transversal, que representa a artéria comunicante anterior. Os dois ramos da artéria carótida interna, logo atrás da origem da futura artéria cerebral posterior, fundem-se em um tronco único mediano, o tronco basilar. Já no estágio final, a parte anterior do ramo caudal da artéria carótida interna sofre uma atrofia, sendo que a artéria vertebral aumenta de volume e supre assim a artéria basilar, onde o sangue passa então a circular de baixo para cima, acontecendo o mesmo com seus ramos de bifurcação anterior (artérias cerebrais anteriores) que parecem continuá-la. Estas informações sugerem que, nos gatos deste estudo, a presença da artéria comunicante rostral, em $46 \%$ dos animais, e ainda contribuição do sistema vertebro-basilar na constituição da porção caudal do circuito arterial do encéfalo

\section{Agradecimentos}

À Faperj e ao CNPq.

\section{Referências}

ALCÂNTARA, M. A.\& I. L. S. PRADA. Arteries of the basis of encephalon in dogs (canis familiares, Linnaeus, 1758). I. Anatomical study of sources and behavior. Brazilian journal of veterinary Research and Animal Sciencie,v. 33, n. 2, p. 67-71, 1996 a

ALCÂNTARA, M. A.; PRADA, I. L. S. Arteries of the basis of the encephalon in dogs (Canis familiaris, Linnaeus, 1758). II. Formation and behaviour of the encephalon arterial circuit . Brazilian journal of veterinary Research and Animal Sciencie, v. 33, n. 2, p. 72-76, 1996 b.

AL SAGAIR, O.; EL MOUGY, S.A. A comparative morphometric study on the skull of three phenotypes of (camels dromedaries), Journal of Camel Practice and Research., v. 9 n. 1, p. 73-77, 2002.

BARONE R.; BORTOLAMI, R. .Anatomie comparée des Mammifères domestiques

Tome 6: Neurologie I. Système nerveux central.Paris, Vigot Frères éditeurs, 652 p, 2004

BREHM ,V.H; LOEFFLER, K. ; KOMEYLI, H. Schädelformen beim Hund_ Anatomia, Histologia, Embryologia, v. 14, n. 4, p. 324-331,1985.

CUNHA, I.P. et al. Comportamento anatômico da artéria cerebelar média no cão (Canis familiaris, Linnaeus, 1758). Veterinária Notícias, v. 7, n. 2, p. 13-22, 2001.

DE VRIESE, B. Sur la signification morphologique des artères cerebrales. Archives de Biologie, v. 21, p. 357-457, 1905.

EVANS, H. E. Miller's anatomy of the dog, 4. ed . Pliladelphia: W. B. Saunders, 2012787 p.

GETTY, R., Sisson/Grossman. Anatomia dos animais domésticos. 5. ed. Interamericana, Rio de Janeiro, 1134 p. 1986 colocam estes espécimes entre o estágio médio e final de evolução ontogenética destes animais.

Do ponto de vista evolutivo e ainda no que se refere ao significado funcional dos vasos estudados, observamos que os gatos apresentam suas fontes sanguíneas para o encéfalo bastante estruturadas, que se expressa através dos mais variados arranjos anastomóticos. Essa distribuição pode ser vista como mecanismo de segurança, ou mesmo compensatório, direcionando adequadamente o fluxo sanguíneo arterial, por caminhos opcionais, na vigência de processo obstrutivo (Lima et al 2006).

\section{Conclusão}

Machos apresentaram maior índice da face enquanto as fêmeas apresentaram maior índice do crânio. Os machos apresentaram valores de largura do hemisfério cerebral esquerdo maiores que os das fêmeas. O padrão vascular do circuito arterial no gato se enquadra entre os subtipos 2 alfa e 2 beta e encontra-se entre os estágios médio e final de evolução filogenética.

HAYES, H. M.; WILSON Jr ,G. P..; FRAUMENÝ Jr, H. F. Carcinoma of the nasal cavity and paranasal sinuses in dogs: descriptive epidemiology. The Cornell veterinarian, v. 72, n. 2, p. 168-179, 1982.

IGADO, O. O.; AINA, O. O. Some aspects of the neurometrics and oculometrics of the japanese quail (Coturnix coturnix japonica) in Nigéria. Journal_of Morphological Science, v. 27, n. 3- 4, p.133135, 2010

KÜNZEL, W.; BREIT, S.; OPPEL, M. Morphometric investigations of breed-specific features in feline skulls and considerations on their functional implications. Anatomy Histology and Embryology, v. 32 , n. 4 , p. $218-23,2003$

LIMA, E.M.M.; PRADA, I. L. S.; SILVA, F. O. C.; SEVERINO, R. S.; SANTOS, A. L. Q.; DRUMMOND, S. S.; RODRIGUES, G. S. Estudo anatômico das artérias da base do encéfalo em gatos. Ars Veterinaria, v. 22, n. 1, p.1-7, 2006.

LINDEMANN, T.; RECKZIEGEL S.; CAMPOS, R. A systematic study of brain base arteries in the opossum Didelphis albiventris. Brazilian journal of morphological sciences., v.17,n.1, p. 35- 41, 2000.

MENESES, S. M. Neuroanatomia aplicada. 3. ed. Rio de Janeiro: Guanabara Koogan, 2011. 368 p.

MORAES S. O. S.; GOMES, M. S. ; SCHERER, P. O. ; ABIDUFIGUEIREDO, M. Morfometria do encéfalo e formação do circuito arterial em equinos mestiços. Ciência Animal Brasileira (Online), v. 15, p. 330-338, 2014.

ONAR, V. A Morphometric Study on the Skull of the German Shepherd Dog Anatomia, Histologia, Embryologia, v. 28, n. 4, p. 253 - 256, 1999.

OLOPADE, J. O.; ONWUKA, S. K.; BALOGUN, B. A. \& OKE, B. O Morphometric investigation of the brain of West African dwarf sheep in Nigeria. International Journal of Morphology, v. 23, n. 2, p. $99-104,2005$. 
PRADA, I. L. S. Neuroanatomia Funcional em Medicina Veterinária com correlações clínicas. $1^{\text {a }}$ ed. Editora Terra Molhada, 616 p, 2014

PETERS, M.; JANCKE, L.; STAIGER, J. F.; SCHLAUS, G,; HELANG, Y. STEINMETZ, H. Unsolved problems of comparing brain sizes in Homo sapiens, Brain and Cognition, v.37, n. 2 , p. 254-285, 1998.

PORTUGAL, J. O.; MORAES S. O. S.; REIS R. M.; GOMES, M. S.; ABIDU-FIGUEIREDO, M. Morfometria do encéfalo e formação do circuito arterioso em coelhos da Nova Zelândia. Biotemas, v. 27, p. 147-155, 2014.

REGEDON, S.; ROBINA, A.; FRANCO, A.; VIVO, J. M.; LIGNEREUX, Y._Détermination Radiologique et Statistique des Types Morphologiques Crâniens chez le Chien: Dolichocéphalic, Mésocéphalie et Brachycéphalie. Anatomia, Histologia,

Embryologia, v. 20, n. 2, p.129-138. 1991

REIF, J.S.; BRUNS, C. \& LOWE, K.S. Cancer of the nasal cavity and paranasal sinuses and exposure to environmental tobacco smoke in pet dogs. American journal of epidemiology, Baltimore, v.147, n. 5, p. 488-492, 1998.

RIBEIRO, P.J.; CRICENTI, V. S.; LANCELLOTTI, C. L. P. Encefalometria na face medial do hemisfério cerebral humano: estudo em necropsias. Arquivos de Neuro-Psiquiatria , v. 63, n. 2b, 2005.
SAMUEL, O. M.; JAMES O. OLOPADE; ONWUKA, S. K. Neurometric evaluations on the brain of the opossum (didelphys marsupialis cancrivora (linnaeus 1758) - a case for cognitive skill -brain development capacity. Journal of Morphological Science, v. 31, n 3. p. 139-145, 2014

TANDLER, J. Zur vergleichenden anatomie der Kopfarterien bein den Mammalia. Denkschriften Akademie der Wissenschaften in Wien, v .67, p. 677-784, 1898

TESTUT, L. Traité de anatomie humaine. 6. ed. Paris: Octave Doin, 1911.

VORIS, H.C. The arterial supply of the brain and spinal cord of the Virginian opossum (Didelphis virginiana). Journal of Comparative Neurology , v. 44, n. 3, p. 403-423. 1928.

WILSON, G.P.; OLSON, L.E. Canine skull linear and tric morphometrics. Anatomia, histologia, embryologia, v..14, p..162191. 1985.

ZIETZSCHMANN, O.; ACKERCNECHT, E.; GRAU, H.;

ELLENBERGER-BAUM. Handbuch der vergleichenden Anatomie der Haustiere. 18. ed. Berlin: Springer, 1943. p. 679. 